\title{
CONTEÚDO DE ENTROPIA NA FÍSICA DO ENSINO MÉDIO: ANÁLISE DO MATERIAL DIDÁTICO E ABORDAGEM HISTÓRICA
}

\author{
Zanoni Tadeu Saraiva dos Santos \\ Professor de Física do CEFET-RN. E-mail: zanoni.tadeu@ cefetrn.br
}

\begin{abstract}
RESUMO
O ensino da segunda lei da termodinâmica e do seu conceito, associado à entropia, tem sido considerado problemático por vários autores. Nesse trabalho iremos compilar os problemas levantados por alguns destes autores na perspectiva de sintetizar as questões e soluções sugeridas, como forma de ampliar a discussão sobre como tratar didaticamente a segunda lei da termodinâmica e a entropia na Física do ensino médio. Fizemos um breve levantamento da abordagem dada ao assunto pelos livros didáticos e propomos que a história e a epistemologia da ciência tenham um papel estruturante em uma prática educativa pautada pelo diálogo. Sugerimos, como parte da construção de um caminho para a entropia, o estabelecimento de três momentos epistemológicos dentro da história da termodinâmica. Esses três momentos serão os balizadores de uma abordagem mais significativa no sentido de que a história da ciência não tenha apenas um papel periférico para o estudo da segunda lei e da entropia.
\end{abstract}

Palavras-chave: Termodinâmica, entropia, ensino, história da ciência.

\section{TEACHING ENTROPY IN SECONDARY PHYSICS: ANALYSIS OF TEXTBOOKS AND A HISTORICAL APPROACH.}

\begin{abstract}
Many authors consider teaching the second law of thermodynamics historically problematic. In this paper we will compile problems raised by some of these authors in the perspective of synthesizing questions and solutions suggested, as a mean to widen the discussion on how to treat didactically the second law and entropy in high school level. We did a brief review on how textbooks approach this subject and came to a conclusion that the history of science and the study of relationships among, science, technology and society should be considered as integrating part of any didactical approach grounded in a dialogical educational view. We suggest as part of a way to entropy, the establishment of three epistemological moments within the history of thermodynamics. These three moments will serve as guiding elements for a more meaningful approach, in a sense that the history of science will not only play a peripheral role in the didactic treatment of entropy and the second law of thermodynamics.
\end{abstract}

Keywords: Thermodynamics, entropy, teaching, history of science. 


\section{CONTEÚDO DE ENTROPIA NA FÍSICA DO ENSINO MÉDIO: ANÁLISE DO MATERIAL DIDÁTICO E ABORDAGEM HISTÓRICA}

\section{INTRODUÇÃO}

Este trabalho nasce no contexto da sala de aula, no trabalho com alunos de licenciatura em Física e alunos de ensino médio do Centro Federal de Ensino Tecnológico do Rio Grande do Norte - CEFETRN. A experiência de ser professor do ensino médio e participar na formação de professores para o mesmo nível de ensino pode ser extremamente rica. É uma oportunidade de transmitir não apenas informação científica, mas também contribuir para a formação destes futuros professores a partir da visão de quem conhece algumas das dificuldades com as quais o futuro professor irá se deparar no seu trato com as disciplinas. Uma dessas dificuldades é construir um tratamento didático que possa ser considerado satisfatório para discutir o conceito de entropia no ensino médio. Discutiremos ao longo deste artigo propostas apresentadas por alguns autores que visam tornar este conceito mais claro e mais significativo para professores e estudantes. A abordagem histórica no ensino de ciências está presente em algumas destas propostas por se tratar de um assunto que vem sendo amplamente discutido no âmbito da pesquisa em ensino de física.

Em nossa tentativa de encontrar a resposta para essa dificuldade, notamos que ela não era exclusividade nossa. Vários autores têm discutido sobre as dificuldades do ensino da termodinâmica de uma maneira geral e da segunda lei e o conceito de entropia de forma específica.

A segunda lei da termodinâmica é uma das construções intelectuais mais intrigantes de todos os tempos. Desde sua primeira formulação no século XIX, tem sido fonte de discussões acaloradas entre cientistas das mais variadas origens, nos mais variados ramos da ciência. Apesar de seu foco ser os sistemas microscópicos, algumas vezes tem sido abusivamente aplicada até mesmo a fenômenos sociais, gerando interpretações que poderíamos classificar, no mínimo, como perigosas. (OLIVEIRA e DECHHOUM, 2003)

De todos os conceitos difíceis da Física Clássica - aceleração, energia, campo elétrico - o mais difícil é entropia. Até mesmo Von Neumann afirmou que "ninguém realmente sabe o que é entropia". (STYER 2000)

A entropia é o assunto mais temido na química universitária básica - por estudantes devido a sua complexidade, e por professores porque os estudantes falham em entender seus princípios básicos. (LAMBERT, 2006)

Os autores manifestam também dificuldades da ordem do entendimento conceitual, ou seja, dificuldades intrínsecas à formulação da segunda lei e do conceito de entropia.

Essas dificuldades parecem residir na falta de correspondência entre o modelo macroscópico das leis da termodinâmica, (relações de energia que empregam grandezas fenomenológicas) e o modelo cinético molecular da matéria. Ambos modelos teóricos, um macroscópico e outro microscópico se apresentam habitualmente na sala de aula, de modo que esta falta de conexão seria em parte proveniente do ensino. (ZAMORANO et alii, 2006)

Entropia não é desordem nem tem nada a ver com coisas misturadas, como cadeiras desarrumadas e cartas embaralhadas. (LAMBERT, 2006) 
A conexão entre a $2^{\mathrm{a}}$ lei e a irreversibilidade é um dos problemas mais profundos da Física. (NUSSENZVEIG, 1981)

A respeito da questão do tratamento estatístico da entropia, Tarsitani e Vicentini (1996) comentam:

Nenhuma fórmula estatística relacionando um conceito indefinido como entropia, com outro conceito indefinido como desordem irá ajudar em um melhor entendimento do conceito de entropia.

Com relação ao uso da metáfora da desordem na explicação do conceito de entropia, Viard (2005) argumenta, baseado em suas pesquisas com alunos de Termodinâmica e física estatística, que este é um "remédio que mata mais do que cura." Em uma amostra de dez alunos, nove associaram entropia a desordem e apenas um foi capaz de responder corretamente a uma questão simples de entendimento do conceito. De forma geral, diz o autor, os alunos associam entropia à desordem espacial e esquecem a parte cinética da entropia.

A abordagem para como tratar didaticamente a segunda lei da termodinâmica e seu conceito associado de entropia, sugerida por Viard (2005), propõe que sejam feitas as perguntas as quais considera fundamentais sobre este conceito. Estas perguntas são aquelas lançadas por Carnot e Clausius e como estes últimos tentaram responder:

O que é esta coisa desconhecida que aparece no campo da ciência, que analisa as relações entre propriedades térmicas e mecânicas dos corpos de acordo com Maxwell quando ele define termodinâmica?

- Por que é necessário introduzir uma nova grandeza física?

Na nossa prática como professor, a partir da forma como a termodinâmica é apresentada nos livros didáticos, chegamos a perguntas semelhantes:

- É o conceito de entropia, do ponto de vista explicativo, estritamente necessário no processo de ensino da termodinâmica básica?

- Sob qual aspecto deve ser tratado, microscópico, macroscópico ou meramente fenomenológico?

- A abordagem macroscópica como tratada por Clausius é adequada ao ensino básico de Física? Por quê? Como resolver a questão do sentido físico da grandeza entropia?

Estas perguntas nos levaram à necessidade de um trabalho mais sistematizado, um projeto de pesquisa que desencadeou nosso ingresso no doutorado em educação. Sentimos a necessidade de construir uma reflexão mais elaborada que pudesse ajudar a encontrar respostas e caminhos não só para nosso trabalho docente, mas também para contribuir à formação dos professores e futuros professores desta área de ensino.

Neste sentido, estruturamos um processo de construção da pesquisa que teve inicio com a análise dos livros didáticos de Física ${ }^{1}$ do nível médio, procurando verificar como estes livros

1 Foram incluídos entre os livros pesquisados, todos os recomendados pelo PNLEM - Programa do Livro Didático do Ensino Médio (04) além do PSSC e GREF, dois títulos com filosofias de ensino de física bem definidas. 
apresentam o conteúdo da termodinâmica, tentando identificar elementos comumente relacionados ao bom desenvolvimento didático de uma disciplina tais como:

a) Se a forma de apresentação do conteúdo tem relação com o seu desenvolvimento histórico. No caso da termodinâmica, se o caráter peculiar ${ }^{2}$ de seu desenvolvimento histórico foi observado.

b) Se existe uma seqüência epistemologicamente coerente de apresentação dos conteúdos. No caso da termodinâmica, se há superposição de visões macroscópicas e microscópicas na explicação dos conceitos.

c) Se o texto usa elementos da tecnologia acessíveis ao aluno como estratégia explicativa dos conceitos. Por exemplo, se é feita uma relação entre uma máquina real e a máquina de Carnot, como forma de explicar a segunda lei da termodinâmica e o conceito de entropia.

\section{Tabela 1 - Abordagem dada à termodinâmica por livros-textos de física do ensino médio.}

\begin{tabular}{|l|c|c|c|c|c|c|}
\hline \multirow{2}{*}{ LIVRO } & \multicolumn{3}{|c|}{$\begin{array}{c}\text { Abordagem predominante da } \\
\text { Termodinâmica }\end{array}$} & \multicolumn{3}{c|}{ Abordagem do conceito de Entropia } \\
\cline { 2 - 7 } & Micro/CM & Macro/LG & Hist/Tec & Macro/FN & Micro/DES & Carnot \\
\hline Penteado & & $\mathrm{X}$ & & & $\mathrm{X}$ & \\
\hline $\begin{array}{l}\text { Beatriz } \\
\text { Alvarenga }\end{array}$ & & $\mathrm{X}$ & $\mathrm{X}$ & & $\mathrm{X}$ & \\
\hline $\begin{array}{l}\text { Alberto } \\
\text { Gaspar }\end{array}$ & & $\mathrm{X}$ & $\mathrm{X}$ & & $\mathrm{X}$ & \\
\hline $\begin{array}{l}\text { Sampaio \& } \\
\text { Calçada }\end{array}$ & & $\mathrm{X}$ & & & $\mathrm{X}$ & \\
\hline Toscano & & $\mathrm{X}$ & & & $\mathrm{X}$ & \\
\hline PSSC & $\mathrm{X}$ & & & & & \\
\hline GREF & & $\mathrm{X}$ & $\mathrm{X}$ & $\mathrm{X}$ & & \\
\hline
\end{tabular}

Legenda da Tabela 1

Micro/CM - abordagem microscópica baseada na teoria cinético-molecular da matéria.

Macro/LG - abordagem microscópica / Lei dos Gases.

His/Tec - abordagem fundada em elementos históricos e da tecnologia.

Macro/FN - abordagem macroscópica fenomenológica

Micro/DES - abordagem microscópica que associa entropia a desordem. Carnot - abordagem que utiliza o ciclo de Carnot como ponto de partida explicativo.

\footnotetext{
${ }^{2}$ Este caráter peculiar se refere ao fato de que o principal produto tecnológico, a máquina a vapor, precedeu o desenvolvimento teórico da disciplina.
} 
A partir dessa análise, sumarizada na tabela 1, verificamos a predominância quase absoluta da abordagem macroscópica para as leis da termodinâmica. A $2^{\mathrm{a}}$ lei é majoritariamente explicada em termos da irreversibilidade das trocas de calor ou da impossibilidade de uma máquina térmica transformar todo calor que recebe em trabalho. No entanto, para o conceito de entropia, há um "salto" epistemológico entre dois enfoques principais: o primeiro trata apenas da irreversibilidade de alguns processos físicos, inclusive das trocas de calor e é feita uma menção de que existe um conceito que expressa essa irreversibilidade, chamado entropia, com o complemento de que, se o processo é irreversível, ocorre um aumento de entropia. $\mathrm{O}$ segundo enfoque é uma tentativa de fazer tratamento microscópico com elementos estatísticos, associando o conceito à desordem dos sistemas. Quanto maior a desordem do sistema, maior a entropia. Vale salientar que, com exceção de dois textos, o tratamento teórico se resume a exemplos qualitativos do tipo "coisas misturadas", ou seja, a entropia é associada meramente a uma desorganização espacial das coisas e não há referência a estados energéticos.

Embora, do ponto de vista histórico, se considere a máquina de Carnot como a fonte teórica da segunda lei $^{3}$, não se encontra nos textos uma relação clara entre esta máquina e o conceito de entropia. O conceito de entropia aparece de forma descontextualizada, isto é, sem relação clara com o funcionamento das máquinas térmicas ou processos termodinâmicos mais gerais. Por exemplo, os livros não esclarecem qual a necessidade teórica do conceito de entropia nos termos macroscópicos fundados por Clausius. Qual a relação entre entropia e energia que nos faria ter a necessidade teórica de criar o novo conceito? Onde exatamente vemos a entropia influenciar nos motores dos automóveis? Exprimir o conceito de entropia apenas como "medida da desordem" é superficial, impreciso, confuso e até mesmo incorreto.

O "salto" que queremos aqui salientar é que a maioria dos livros textos analisados faz um percurso didático essencialmente macroscópico da termodinâmica até à segunda lei. Então, repentinamente, há uma predominância quase total da explicação microscópica para o caso da entropia. Existe uma razão didaticamente coerente para isto, ou é apenas uma maneira cômoda de dar uma explicação de algo complicado?

Com relação ao aspecto do desenvolvimento histórico da termodinâmica, nos referimos ao fato do aparecimento das máquinas a vapor precederem o seu desenvolvimento teórico. $\mathrm{Na}$ figura 1 temos a representação de uma linha do tempo para alguns acontecimentos relevantes da termodinâmica. O fato mais característico do que chamamos de caráter peculiar da história da termodinâmica é que o livro no qual Carnot coloca a questão fundamental sobre o limite de rendimento das máquinas térmicas é publicado quase 100 anos depois do aparecimento das primeiras máquinas a vapor. Toda a revolução econômica e social desencadeada pela máquina de Watt aconteceu sem que se tivesse formalizado os princípios teóricos fundamentais como o da conservação da energia ou a equivalência entre calor e trabalho mecânico.

\footnotetext{
3 A máquina de Carnot impõe um limite teórico ao rendimento das máquinas térmicas o que implica na impossibilidade da transformação total de calor em trabalho numa máquina cíclica e este vem a ser um dos enunciados da segunda lei da termodinâmica.
} 


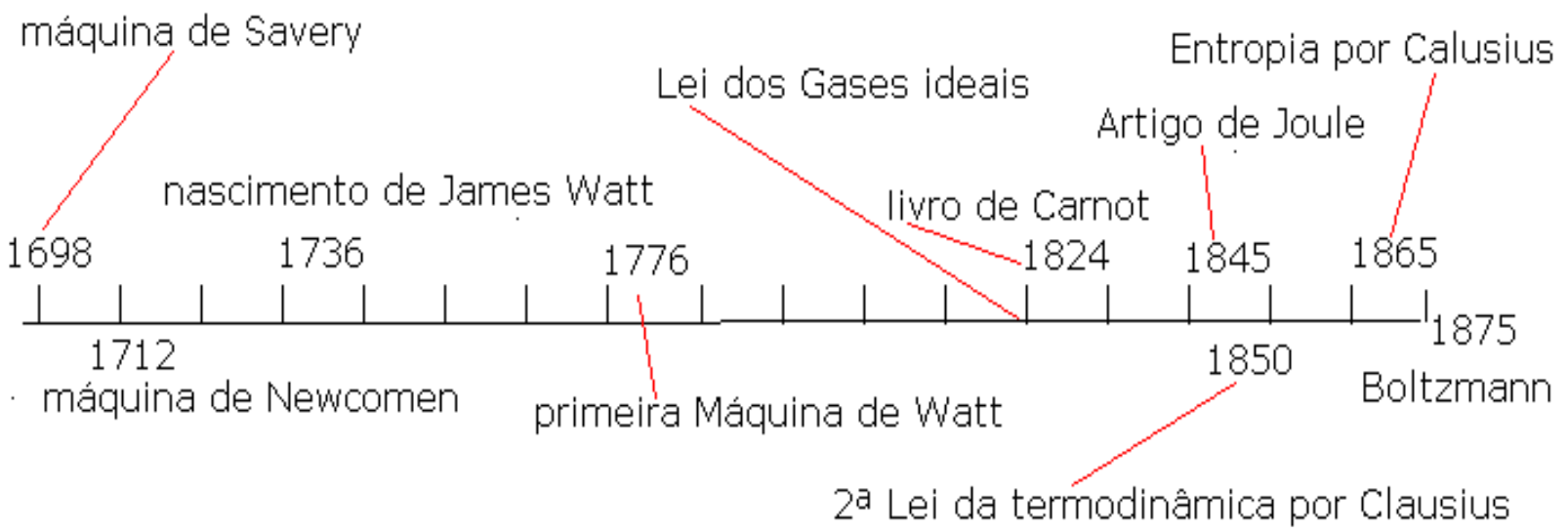

Fig.01- Linha do tempo dos principais eventos da história da termodinâmica

A seguir mostramos, na tabela 2, uma seqüência típica dos conteúdos como aparecem nos livros didáticos e as datas de suas respectivas elaborações e/ou descobertas, em contraposição à linha do tempo mostrada na figura 1 . Mesmo considerando que um conteúdo não deve necessariamente ser apresentado em estrita seqüência histórica, vale a pergunta de qual a razão de tal seqüência.

Tabela 2 - Seqüência típica de conteúdos da termodinâmica nos livros didáticos de Física.

\begin{tabular}{|l|l|}
\hline 1930 & $\begin{array}{l}\text { Lei zero da termodinâmica - tem a função de fundamentar o conceito de } \\
\text { temperatura, mas este é também explicado como relativo à energia cinética das } \\
\text { moléculas. }\end{array}$ \\
\hline 1800 & $\begin{array}{l}\text { Lei dos gases ideais: descreve macroscopicamente o comportamento térmico } \\
\text { dos gases como forma de introduzir a idéia de trabalho realizado por um gás. }\end{array}$ \\
\hline $\begin{array}{l}\text { Equivalente mecânico do calor, energia térmica: trata o calor como uma forma } \\
1847\end{array}$ & $\begin{array}{l}\text { Equivale } \\
\text { de energia, faz a descrição da experiência de Joule sem referência à primeira } \\
\text { Lei. }\end{array}$ \\
\hline 1780 & $\begin{array}{l}\text { Calor específico, calor latente, capacidade térmica são aqui apresentados com } \\
\text { uma linguagem que remete ao calor como um fluido ( calórico). O objetivo } \\
\text { neste tópico é basicamente medir “ a quantidade de calor”. }\end{array}$ \\
\hline 1847 & $\begin{array}{l}\text { Primeira lei da termodinâmica: tem o objetivo de definir o conceito de energia } \\
\text { interna e o principio da conservação da energia. }\end{array}$ \\
\hline 1850 & $\begin{array}{l}\text { Segunda lei da termodinâmica: trata basicamente da impossibilidade de } \\
\text { transformar integralmente calor em trabalho numa máquina térmica. }\end{array}$ \\
\hline 1700 & $\begin{array}{l}\text { Máquinas térmicas: descreve-se o princípio de funcionamento das máquinas } \\
\text { térmicas e os seus ciclos de transformação e calor em trabalho. Exemplo típico, } \\
\text { o motor de combustão interna do ciclo Otto. }\end{array}$ \\
\hline 1824 & Ciclo de Carnot \\
\hline 1875 & Interpretação estatística da Entropia. \\
\hline
\end{tabular}

A sequiência apresentada nos livros didáticos, na qual há idas e vindas no tempo, poderia indicar uma ordem epistemológica coerente. Entretanto, não é o que nos parece para a maioria dos livros. Os textos do GREF e PSSC assumem antecipadamente suas posições epistemológicas: o GREF assume os postulados da teoria cinética molecular clássica e o 
PSSC, a posição estritamente estatística. Entretanto, o primeiro negligencia uma explicação mais aprofundada da entropia e o segundo uma relação com os objetos tecnológicos e a contextualização histórica.

Da mesma forma que quase a unanimidade dos livros didáticos de Física fazem uso de uma associação entre entropia e "desordem", as propostas pedagógicas para um melhor entendimento da segunda lei da termodinâmica e da entropia partem da crítica a essa associação. Os artigos normalmente apresentam situações físicas em que a metáfora da desordem não se aplica para em seguida lançarem suas propostas.

Lambert (2002) chama a desordem de "a muleta quebrada para sustentar a entropia" e que esse foi acima de tudo um artifício elaborado para a visualização e não uma causa física ou teórica fundamental para um maior valor da entropia. Assegura que a natureza da entropia é mais bem ensinada a partir da descrição da sua dependência da dispersão da energia (na termodinâmica clássica) e pela distribuição de energia entre um enorme número de movimentos moleculares relacionados a microestados (na termodinâmica molecular). A variação da entropia é uma propriedade dependente da energia. Esta é sua essência, diz Lambert.

Viard (2005) inicia argumentando que os professores de Física normalmente, em suas explicações, partem de uma palavra para um conceito. Isto não funciona bem para a entropia visto que o termo não se compara a nenhum outro termo conhecido como a palavra resistência aplicada ao conceito de resistência elétrica. No caso da entropia, recorre-se à metáfora da "desordem" como estratégia explicativa o que, segundo o autor, é um remédio que mata mais do que cura.

A proposta lançada por Viard é que alunos e professores percorram a "estrada para a entropia" traçada em trechos escolhidos dos textos originais de Carnot e Clausius nos quais, passo a passo, vão sendo analisados os argumentos que levam a uma grandeza física necessária dentro do contexto da $2^{\mathrm{a}}$ lei. $\mathrm{O}$ autor recupera, através desse caminho, "uma teoria quantitativa das transformações" formulada por Clausius.

A questão agora é encontrar a lei de acordo com a qual nós possamos representar matematicamente estas transformações, de modo que sua equivalência resulte da igualdade de seus valores. Podemos chamar o valor matemático de uma transformação de valor de equivalência." (Clausius 1864)

Esta grandeza é expressa então por Q/T e a segunda pergunta que o autor tenta responder é qual o significado físico deste quociente. A resposta encontrada refere-se ao trabalho realizado pelo calor na desagregação das partículas do corpo (gás). A parte do calor que é transmitida de um corpo a outro sem a realização de trabalho não é abordada.

Oliveira e Dechoum (2003) fazem uma revisão da segunda lei e da máquina de Carnot como forma de esclarecer pontos teóricos importantes. A entropia não é discutida enquanto conceito. Os autores partem da equação consagrada por Clausius (dS=dQ/T) (1) e o objetivo é mostrar que o ensino da segunda lei se torna mais claro com o uso do diagrama (T x S) o que não substitui as construções baseadas no diagrama $(P \times V)$ que $o$ estudante se depara antes de aprender o conceito de entropia. Segundo os autores, "enormes ganhos didáticos como o rendimento de qualquer máquina térmica reversível é visualizado claramente." 
Baierlein (1994) propõe uma inversão temporal partindo da abordagem microscópica /estatística e chegando à equação (1). Adota analogias e exemplos específicos e chega à conclusão de que "não há nada de errado em se referir a entropia como medida da desordem desde que se tenha o cuidado de relacionar desordem com falta de correlação e, portanto, com multiplicidade de estados". Segundo o autor, é a multiplicidade que tem precisão suficiente para servir como base para uma teoria física.

A abordagem feita por Tarsitani e Vicentini (1996) envolve a construção de mapas conceituais dos principais livros-textos usados em cursos universitários básicos. Essa análise tem como propósito analisar os livros sob três critérios: 1) a arquitetura conceitual do livro e a inter-relação entre os princípios (postulados) e conceitos fundamentais. 2) a relação entre o aporte fenomenológico da termodinâmica e o aporte microscópico e 3) o desenvolvimento histórico dos próprios textos didáticos que evidenciariam não apenas um aspecto histórico da termodinâmica, mas principalmente o desenvolvimento didático da disciplina. O objetivo é identificar similaridades e diferenças entre a abordagem epistemológica dos diversos textos. Por estes autores ficamos sabendo que a forma mais comum de arquitetura conceitual dos livros didáticos é derivada do texto de Planck, "Tratado sobre Termodinâmica". Esta arquitetura não faz nenhuma referência ao mundo microscópico e, segundo os autores, Planck acreditava que a entropia é um conceito tão fundamental quanto a energia e deve ser entendida com base na evidência fenomenológica.

A nossa atenção está voltada para a conclusão dos autores de que a maior parte dos livros que apresentam arquiteturas conceituais fenomenológicas (incluindo o Theory of Heat de Maxwell) apresenta a hipótese da estrutura atômica como um artifício conceitual.

A hipótese da estrutura particulada da matéria é usada meramente como ferramenta intuitiva para justificar a existência de variáveis macroscópicas extensivas como energia interna e entropia. (TARSITANI e VICENTINI, 1996)

Voltemos, pois, ao ponto de partida. O conceito de entropia foi estabelecido sem a necessidade da abordagem microscópica, o que não justifica a prevalência de explicações microscópicas e consequentemente a metáfora de desordem. Partilhamos do entendimento de Planck quanto ao status dos conceitos de entropia e energia. Desejamos, com o desenvolvimento desta pesquisa, propor elementos para o tratamento didático da entropia no ensino médio que auxiliem na compreensão da importância deste conceito no corpo teórico da termodinâmica clássica. A partir daí então pretendemos fazer a ponte para a interpretação estatística moderna.

\section{CONSIDERAÇÕES FINAIS}

A proposta que apresentamos como tese tem semelhança metodológica com a de Viard (2005) no sentido de construir uma "estrada para a entropia". Entretanto, a estrada aqui proposta não seria construída apenas sob um ponto de vista histórico, mas teria em sua base "momentos epistemológicos" bem definidos e internamente consistentes. Entendemos que a maior parte dos problemas enfrentados no ensino da termodinâmica tem sua nascente na "salada epistemológica" apresentada pelos livros didáticos e seguida pelos professores. A construção de uma estrada para a entropia com vias epistemológicas definidas e apropriadas para níveis de ensino ou finalidades didáticas específicas, nos parece, não foi ainda devidamente considerada. 
Um primeiro momento desta abordagem didática seguiria claramente a linha do tempo e seria chamado de momento técnico-substancialista. Nele, basicamente, nos interessaria o processo técnico de construção e funcionamento das máquinas térmicas. Acreditamos não haver nenhum impedimento teórico em tratar este momento de rendimento de máquinas térmicas e ciclo de Carnot anteriormente a qualquer noção microscópica de calor e temperatura.

O segundo momento seria o do calor como processo e transformação. Define-se aqui a natureza do calor com base na definição da energia interna e sua relação com trabalho. Neste ponto o calor deixa de ser uma "substância sutil", perde sua materialidade e se converte em um processo que só pode ser verificado através dos seus efeitos. Consideramos que o conceito de energia, fortemente associado à idéia de processo e transformação, seria o aspecto estruturante deste momento, em lugar das tentativas de "conceituação" da energia como grandeza, quase sempre imprecisas e vagas. Abre-se assim uma porta para a introdução do conceito de entropia porque ele está epistemologicamente associado à equivalência dessas transformações.

Clausius definiu a entropia, a princípio, sem a necessidade de nenhum argumento microscópico, tendo como idéia principal a equivalência das transformações. Em uma transformação reversível, as grandezas físicas envolvidas assumem valores iguais e simétricos em ambos os sentidos do processo. Nesse caso deve haver uma grandeza que nos informe dessa reversibilidade por meio de uma soma algébrica nula de seu valor em um sentido e no outro.

O terceiro e último momento seria o da Teoria cinético-molecular que faria a ponte entre a termodinâmica clássica e sua abordagem estatística.

Queremos chamar a atenção neste trabalho para o fato de que a história da termodinâmica pode ter uma participação fundamental como elemento explicativo dos conceitos aqui considerados. Levantar aspectos históricos do conhecimento científico nos parece um procedimento que não pode ser tratado de modo periférico, dentro de uma perspectiva dialógica na qual o aluno deve como sujeito do conhecimento, ter participação efetiva no processo. Já nos referimos ao aspecto diferenciado do desenvolvimento da termodinâmica e existem razões históricas para isso que oferecem as condições para o aprimoramento da discussão tanto dos aspectos científicos quanto sociais que permeiam o ambiente do ensino das ciências. 


\section{REFERÊNCIAS}

Alvarenga, Beatriz; Máximo, Antônio. Física. Vol 2. São Paulo: Scipione, 2007.

Aurélio G. Filho; Carlos Toscano. Física. São Paulo: Scipione, 2007.

Baierlein, Ralph. Entropy and the second Law: a pedagogical Alternative, American Journal of Physics, ano 62 vol. 1, p.15-26. 1994.

Gaspar, Alberto. Física. São Paulo: Scipione, 2005.

GREF - Grupo de Reelaboração do Ensino de Física. Física Térmica e Óptica 2. $4^{\mathrm{a}}$ ed. São Paulo: Ed. Univ. de São Paulo, 1998.

Lambert, Frank. Disorder - Cracked crutch for Supporting Entropy Discussions. Journal of Chemical Education. Vol 49, nº.2, Feb, 2002. p. 187-192.

Oliveira, P.M.C.; Dechoum, K. Facilitando a compreensão da Segunda lei da Termodinâmica. Revista Brasileira de Ensino de Física. Vol 25, nº4, Dezembro, 2003. p. 359-363.

Penteado, Paulo César; Torres, Carlos Magno. Física Ciência e Tecnologia. Vol 2. São Paulo: Moderna, 2005.

PSSC - COLLEGE PHYSICS - Del Physical Science Study committee. Zaragoza: Editorial Luis Vives, 1969.

Sampaio, José Luiz; Calçada, Caio Sergio. Universo da Física. $2^{\text {a }}$ ed. São Paulo: Ed. Atual, 2005.

Styer, Daniel F. Insight into Entropy. American Journal of Physics, ano 68, vol.12, 2000. p.1090-1096.

Tarsitani C.; Vicentini, M. Scientific Mental Representations of Thermodynamics. Science Education. vol. 5,1996. 51-68.

Viard, Jèrôme. Using the history of Science to Teach Thermodynamics at the University level: The Case of the Concept of Entropy. Disponível em www.ihpst2005.leeds.ac.uk/papers/Viard.pdf , arquivo capturado em 15/12/2007.

Zamorano, Raul O.; Gibbs, Horácio M.; Maio, Lucrecia E; Viau, Javier E. Evaluación de um modelo didáctico analógico para el aprendizaje de energia interna e temperatura. Revista Eureka - sobre enseñaza e divulgación de las ciências. Ano 3, vol.3, 2006. p.392-408. 